\title{
A DISCRETE-TIME SYSTEM WITH SERVICE CONTROL AND REPAIRS
}

\author{
IVAN ATENCIA
}

\author{
Department of Applied Mathematics \\ University of Malaga, Campus de Teatinos, 29071 Malaga, Spain \\ e-mail: iatencia@ctima.uma.es
}

\begin{abstract}
This paper discusses a discrete-time queueing system with starting failures in which an arriving customer follows three different strategies. Two of them correspond to the LCFS (Last Come First Served) discipline, in which displacements or expulsions of customers occur. The third strategy acts as a signal, that is, it becomes a negative customer. Also examined is the possibility of failures at each service commencement epoch. We carry out a thorough study of the model, deriving analytical results for the stationary distribution. We obtain the generating functions of the number of customers in the queue and in the system. The generating functions of the busy period as well as the sojourn times of a customer at the server, in the queue and in the system, are also provided. We present the main performance measures of the model. The versatility of this model allows us to mention several special cases of interest. Finally, we prove the convergence to the continuous-time counterpart and give some numerical results that show the behavior of some performance measures with respect to the most significant parameters of the system.
\end{abstract}

Keywords: discrete-time queue, unreliable server, negative customers, busy period, sojourn times, continuous-time counterpart.

\section{Introduction}

Queueing systems constitute a central tool in modelling and performance analysis. These types of systems are in our everyday life activities, and the theory of queueing systems was developed to provide models for forecasting behaviors of systems subject to random demand.

Most queueing models in the literature before the early 1990s were developed in continuous time and only the models that were based on embedded Markov chains were studied in discrete times, e.g., models such as $M / G / 1$ and $G I / M / 1$, which were well discussed in the 1950s by Kendall (1951a; 1951b).

During the last few decades, there has been a keen interest in the study of discrete-time queueing systems. One of the main reasons is that discrete-time systems are more suitable than their continuous-time counterparts for modelling computer and telecommunication systems, since the basic units in these systems are digital, such as machine cycle times, bits and packets. The modelling of a communication system is an area that uses queueing models significantly, and continuous time models were seen as adequate for the purpose. However, the communication systems are now more digital than analogical and we work in time slots. Therefore, discrete modelling has become more appropriate. Another use of discrete-time models is to approximate continuous-time models via time quantization, which especially serves for the numerics of direct computations (see Kleinrock, 1976; Yang and Li, 1995).

The pioneering work on discrete-time queueing system was carried out by Meisling (1958). Reference works and more detailed applications on discrete-time queueing theory include the monographs by Bruneel and Kim (1993) as well as Takagi (1993). Further, a detailed treatment regarding this subject can be found in a two-volume book on applied probability by Hunter (1983) and in the one by Alfa (2010).

In most of the queueing literature, it is assumed that the server is always available, but this assumption is practically unrealistic. A notable and inevitable phenomenon in the service facility of a queueing system is its breakdown and consequent repair. Until the server facility is recovered again, the waiting time for customers will increase with a consequence effect over the impatience of the customer. Indeed, queueing systems with server breakdowns are very common in communication systems. Queues with service interruptions were first studied by White and 
Christie (1958). In queueing theory parlance, periods of temporary service unavailability are referred to as server vacations, server interruptions or server breakdowns. Vacation queues are a very important class of queues because of their theoretical structure as well as their applicability in various real life situations, especially in telecommunication systems where Medium Access Control (MAC) is a critical component of managing a successful network. A vacation is the period of not attending a certain task, but this is due to the fact that the server is under repair, attending other queues, or in priority queues where a low priority customer receives service until a higher priority one arrives. In this case the server may switch off. That is, a lower priority customer will see the server as on vacation. Vacation models have been used extensively to study various systems, such as polling and some priority ones.

This work analyses a discrete-time queue with the service station subject to starting failures. It is assumed the breakdowns take place in accordance with the preemptive non-resume strategy, i.e., the customer whose services are unsuccessful waits in the queue and, when the service resumes for the preempted customer, it starts again from the beginning.

Developing analytical models to be used for analyzing their performance is a very important issue, which has been dealt with by several researchers. Most of the existing models focus on continuous-time ones (e.g., Aissani and Artalejo, 1998; Artalejo, 1994; Krishna Kumar et al., 2002; Kulkarni and Choi, 1990; Yang and Li, 1994; Krishnamoorthy et al., 2009) and application to networks with blocking and truncation (Oniszczuk, 2009). Works related to discrete-time systems with server interruptions with or without expulsions and vacations can be found, including those by Fiems et al. (2002; 2004), Vinck and Bruneel (2006), Morozov et al. (2011) as well as Atencia and Pechinkin (2012) and Atencia et al. (2013a; 2013b).

Performance prediction in communication switching queues, job processing in computers, etc., are always influenced by customers' behaviour, and the provision of this additional information will be useful in upgrading the service. Therefore, in many real problems it is also interesting to consider the movement of jobs, customers, etc. from one place to another. This mechanism is called a synchronized or triggered motion (e.g., Artalejo, 2000; Gelenbe and Label, 1998). For the inverse order discipline, we refer to Pechinkin and Svischeva (2004), Pechinkin and Shorgin (2008) as well as Cascone et al. (2011).

The arrival of a negative customer to a queueing system causes one ordinary customer to be removed or killed, if any is present. Pioneering work on discrete-time considering negative arrivals without retrials was done by Atencia and Moreno (2004; 2005), who considered several killing strategies for negative customers. For a survey on this topic, see Gelenbe and Label (1998) or Artalejo (2000), for applications in engineering refer to Chao et al. (1999), and for applications in communication networks and packet transmission systems see Harrison et al. (2000), Park et al. (2009) as well as Piórkowski and Werewka (2010).

In order to design a queueing system, we need to find configurations and rules that will optimize it. To do this, we need to understand how the queueing system will perform under different configurations and rules like the queue length, which refers to the number of items or customers waiting in some location or buffer in order to be processed. This is often a good indicator on how a queueing system is performing. The longer the queue length, the worse its performance from the user's point of view. The waiting times or the delay for a customer to wait until getting service are another measure of the behavior of the system. Of course, the longer this performance measure, the worse the perception of the system from the customer's point of view in terms of the service time. The busy period is also an interesting measure. This measure is more of interest in order to keep his/her resources fully utilized, for example, in ISPs (Internet Service Providers). However, if the resource that is used to provide service is human, such as in a bank, store, etc., then there is a limit on how long a service provider wishes to keep a server busy before it becomes ineffective. Finally, we point out that the objective of this work is to obtain all important performance measures for a rather versatile discrete-time queueing model by means of a complete analysis that allow us to obtain several previously found results as special cases.

The rest of this paper is organized as follows. The next section gives a description of the queueing model. In Sections 3 and 4, we study the Markov chain and the stability condition of the system. The queue and system size distributions are also obtained, together with several performance measures. In Section 5 we study the stationary distribution of the busy period. In Section 6, we present the stationary distributions of the sojourn times of a customer in the server and in the system. In Section 7, we show how the continuous-time version of our model can be approximated by its discrete-time counterpart. Finally, in the last section, some numerical examples are presented.

\section{Mathematical model}

Discrete-time queueing models have been widely used over the past years in view of their applicability in the study of many computer and communication systems. In this type of systems, the time axis is partitioned into intervals of equal length, called slots. It should be pointed out that the probability of an 
arrival and a departure occurring simultaneously is zero in continuous-time queues, whereas it is not so in discrete-time systems. Since more than one different event may occur concurrently, to resolve conflicts it is necessary to establish the order in which the arrivals, departures and repairs take place in the case of simultaneity. Basically, there are two rules:

(i) If an arrival takes precedence over a departure, it is identified with a Late Arrival System (LAS) (see Fig. 1(a)).

(ii) If a departure takes precedence over an arrival, it is recognized by an Early Arrival System (EAS) (see Fig. 1(b)).

The former case is also known as the Arrival First (AF) policy and the latter as the Departure First (DF) policy. For more details on these and related concepts, see Gravey and Hébuterne (1992) as well as Hunter (1983). In the present paper, we will follow the second policy.

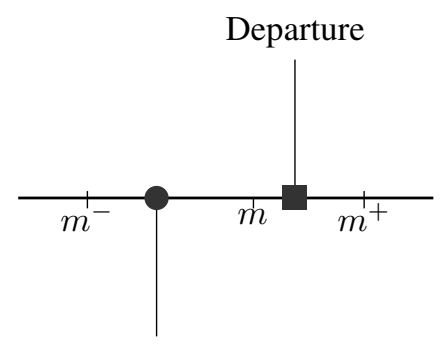

Arrival

(a)

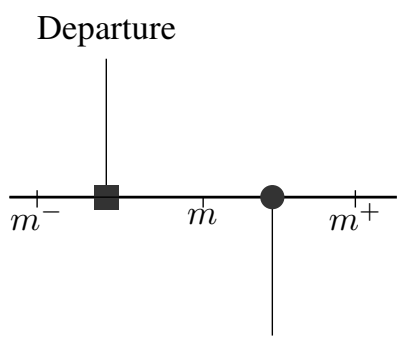

Arrival

(b)

Fig. 1. Options of the arrival models.

Therefore, let the time axis be marked by $0,1,2, \ldots, m, \ldots$. Consider the epoch $m$ and suppose that the end of services and repairs occur in $\left(\mathrm{m}^{-}, \mathrm{m}\right)$, and the arrivals and the beginning of the repairs in $\left(m, m^{+}\right)$.

In this paper we consider a discrete-time queueing system with starting failures in which an arriving customer follows three different strategies. The first and the second follow the LCFS discipline with displacements and expulsions, respectively. The third one behaves itself as a negative customer, that is, expels out of the system the customer that is currently being served and has no further influence on the system. Customers arrive according to a Bernoulli arrival process with rate $a$, that is, $a$ is the probability that a customer arrives at a slot. A customer who enters the server must turn on the service station. If the server is activated successfully (with probability $\gamma$ ), the customer begins his/her service immediately and if the server at the moment of his/her arrival is busy, with probability $\theta_{1}$ displaces the customer that was currently being served to the first place of the queue, with probability $\theta_{2}$ expels out of the system the customer that was in service, and with probability $\theta_{3}$ the arriving customer becomes a negative customer, that is, kills the customer in service and has no effect on an empty system; otherwise, if the server is started unsuccessfully (with complementary probability $\bar{\gamma}$ ), it is sent to repair directly and the customer is placed at the head of the queue. The customers that arrive to the system during a repair time go directly to the last place of the queue. Clearly, $\theta_{1}+\theta_{2}+\theta_{3}=1$.

The service times are independent and identically distributed with a general distribution $\left\{s_{1, i}\right\}_{i=1}^{\infty}$ and a generating function

$$
S_{1}(x)=\sum_{i=1}^{\infty} s_{1, i} x^{i} .
$$

Certainly, after service completion, the served customer leaves the system forever and will have no further effect on the system.

The repair times are independent and identically distributed with an arbitrary distribution $\left\{s_{2, i}\right\}_{i=1}^{\infty}$ with a generating function

$$
S_{2}(x)=\sum_{i=1}^{\infty} s_{2, i} x^{i},
$$

and $n$-th factorial moments $\beta_{2, n}$. Naturally, after repair, the service station is as good as new.

The load of the system is given by $\rho_{1}+\rho_{2}$, where

$$
\begin{aligned}
& \rho_{1}=a\left(1-\theta_{3}\right) \bar{b}, \\
& \rho_{2}=\frac{a \bar{a} \bar{\gamma}\left(1-\theta_{3}\right) \beta_{2,1}}{\gamma\left[\theta_{1} S_{1}(\bar{a})+\bar{a}\left(1-\theta_{1}\right)\right]},
\end{aligned}
$$

and $\bar{b}$ is the mean sojourn time of a customer in the server.

For most times in queueing applications, Markov chains are more common even though continuous space Markov processes, in the form of stochastic fluid models, are getting popular with applications to queueing systems. Therefore, our queueing model has been reduced to a Markov chain analysis, as otherwise it would be difficult to solve it. 


\section{Markov chain}

At time $m^{+}$, the system can be described by the process

$$
\left(C_{m}, \xi_{1, m}, \xi_{2, m}, N_{m}\right),
$$

where $C_{m}$ denotes the state of the server, 0,1 or 2 according to whether the server is free, busy or down, and $N_{m}$ is the number of customers in the queue. If $C_{m}=1$, $\xi_{1, m}$ represents the remaining service time of the customer currently being served, and if $C_{m}=2, \xi_{2, m}$ corresponds to the remaining repair time.

It can be shown that $\left\{\left(C_{m}, \xi_{1, m}, \xi_{2, m}, N_{m}\right), m \in\right.$ $\mathbb{N}\}$, provides a Markovian description of our queueing system, whose state space is

$$
\begin{gathered}
\chi=\{(0),(1, i, k): i \geq 1, k \geq 0 ; \\
(2, i, k): i \geq 1, k \geq 1\} .
\end{gathered}
$$

Our first objective is to find the stationary distributions

$$
\pi_{0}=\lim _{m \rightarrow \infty} P\left[C_{m}=0\right],
$$

of the Markov chain $\left\{\left(C_{m}, \xi_{1, m}, \xi_{2, m}, N_{m}\right), m \in \mathbb{N}\right\}$. For $i \geq 1, k \geq 0$ we have

$$
\pi_{1, i, k}=\lim _{m \rightarrow \infty} P\left[C_{m}=1, \xi_{1, m}=i, N_{m}=k\right],
$$

and for $i \geq 1, k \geq 1$ we get

$$
\pi_{2, i, k}=\lim _{m \rightarrow \infty} P\left[C_{m}=2, \xi_{2, m}=i, N_{m}=k\right] .
$$

The evolution of the chain is governed by the one-step transition probabilities given by

$$
\begin{aligned}
p_{(0)(0)} & =\bar{a}+a \theta_{3}, \\
p_{(1,1,0)(0)} & =\bar{a}+a \theta_{3}, \\
p_{(1, i, 0)(0)} & =a \theta_{3}, \quad i \geq 2 .
\end{aligned}
$$

If $i \geq 1$ and $k \geq 0$ we have

$$
\begin{aligned}
p_{(0)(1, i, k)} & =a\left(1-\theta_{3}\right) \gamma s_{1, i}, \quad k=0, \\
p_{(1,1, k)(1, i, k)} & =a\left(\theta_{1}+\theta_{2}\right) \gamma s_{1, i}, \\
p_{(1,1, k+1)(1, i, k)} & =\left(\bar{a}+a \theta_{3}\right) \gamma s_{1, i}, \\
p_{(1, i+1, k)(1, i, k)} & =\bar{a}+a \theta_{2} \gamma s_{1, i}, \\
p_{(1, j, k-1)(1, i, k)} & =a \theta_{1} \gamma s_{1, i}, \quad j \geq 2, \\
p_{(1, j, k)(1, i, k)} & =a \theta_{2} \gamma s_{1, i}, \quad j \geq 2, \quad j \neq i+1, \\
p_{(1, j, k+1)(1, i, k)} & =a \theta_{3} \gamma s_{1, i}, \quad j \geq 2, \\
p_{(2,1, k)(1, i, k)} & =a\left(1-\theta_{3}\right) \gamma s_{1, i}, \quad k \geq 1, \\
p_{(2,1, k+1)(1, i, k)} & =\left(\bar{a}+a \theta_{3}\right) \gamma s_{1, i} .
\end{aligned}
$$

If $i \geq 1$ and $k \geq 1$, we have

$$
\begin{aligned}
p_{(0)(2, i, k)} & =a\left(1-\theta_{3}\right) \bar{\gamma} s_{2, i}, \quad k=1, \\
p_{(1,1, k-1)(2, i, k)} & =a\left(\theta_{1}+\theta_{2}\right) \bar{\gamma} s_{2, i}, \\
p_{(1,1, k)(2, i, k)} & =\left(\bar{a}+a \theta_{3}\right) \bar{\gamma} s_{2, i}, \\
p_{(1, j, k-2)(2, i, k)} & =a \theta_{1} \bar{\gamma} s_{2, i}, \quad j \geq 2, \quad k \geq 2 \\
p_{(1, j, k-1)(2, i, k)} & =a \theta_{2} \bar{\gamma} s_{2, i}, \quad j \geq 2,
\end{aligned}
$$

$$
\begin{aligned}
p_{(1, j, k)(2, i, k)} & =a \theta_{3} \bar{\gamma} s_{2, i}, \quad j \geq 2, \\
p_{(2,1, k-1)(2, i, k)} & =a\left(1-\theta_{3}\right) \bar{\gamma} s_{2, i}, \\
p_{(2,1, k)(2, i, k)} & =\left(\bar{a}+a \theta_{3}\right) \bar{\gamma} s_{2, i}, \\
p_{(2, i+1, k-1)(2, i, k)} & =a\left(1-\theta_{3}\right), \quad k \geq 2, \\
p_{(2, i+1, k)(2, i, k)} & =\bar{a}+a \theta_{3},
\end{aligned}
$$

where $\bar{a}=1-a$.

The Kolmogorov equations for the stationary distribution of the system are given by

$$
\begin{aligned}
\pi_{0}= & \left(\bar{a}+a \theta_{3}\right) \pi_{0}+\bar{a} \pi_{1,1,0}+a \theta_{3} \sum_{i=1}^{\infty} \pi_{1, i, 0} \\
\pi_{1, i, k}= & \delta_{0, k} a\left(1-\theta_{3}\right) \gamma s_{1, i} \pi_{0}+a \theta_{1} \gamma s_{1, i} \pi_{1,1, k} \\
& +\bar{a} \gamma s_{1, i} \pi_{1,1, k+1}+\bar{a} \pi_{1, i+1, k} \\
& +\left(1-\delta_{0, k}\right) a \theta_{1} \gamma s_{1, i} \sum_{j=2}^{\infty} \pi_{1, j, k-1} \\
& +a \theta_{2} \gamma s_{1, i} \sum_{j=1}^{\infty} \pi_{1, j, k}+a \theta_{3} \gamma s_{1, i} \sum_{j=1}^{\infty} \pi_{1, j, k+1} \\
& +\left(1-\delta_{0, k}\right) a\left(1-\theta_{3}\right) \gamma s_{1, i} \pi_{2,1, k} \\
& +\left(\bar{a}+a \theta_{3}\right) \gamma s_{1, i} \pi_{2,1, k+1}, \quad i \geq 1, k \geq 0
\end{aligned}
$$

$$
\begin{aligned}
\pi_{2, i, k}= & \delta_{1 k} a\left(1-\theta_{3}\right) \bar{\gamma} s_{2, i} \pi_{0}+a \theta_{1} \bar{\gamma} s_{2, i} \pi_{1,1, k-1} \\
& +\left(\bar{a}+a \theta_{3}\right) \bar{\gamma} s_{2, i} \pi_{1,1, k} \\
& +\left(1-\delta_{1 k}\right) a \theta_{1} \bar{\gamma} s_{2, i} \sum_{j=2}^{\infty} \pi_{1, j, k-2} \\
& +a \theta_{2} \bar{\gamma} s_{2, i} \sum_{j=1}^{\infty} \pi_{1, j, k-1} \\
& +a \theta_{3} \bar{\gamma} s_{2, i} \sum_{j=2}^{\infty} \pi_{1, j, k} \\
& +\left(1-\delta_{1 k}\right) a\left(1-\theta_{3}\right) \bar{\gamma} s_{2, i} \pi_{2,1, k-1} \\
& +\left(\bar{a}+a \theta_{3}\right) \bar{\gamma} s_{2, i} \pi_{2,1, k} \\
& +\left(1-\delta_{1 k}\right) a\left(1-\theta_{3}\right) \pi_{2, i+1, k-1} \\
& +\left(\bar{a}+a \theta_{3}\right) \pi_{2, i+1, k}, i \geq 1, \quad k \geq 1,
\end{aligned}
$$

where $\delta_{a, b}$ is Kronecker's symbol and the normalizing condition is

$$
\pi_{0}+\sum_{i=1}^{\infty} \sum_{k=0}^{\infty} \pi_{1, i, k}+\sum_{i=1}^{\infty} \sum_{k=1}^{\infty} \pi_{2, i, k}=1 .
$$

With the aim of solving Eqns. (1) and (2), we introduce the following generating functions:

$$
\begin{aligned}
& \varphi_{1}(x, z)=\sum_{i=1}^{\infty} \sum_{k=0}^{\infty} \pi_{1, i, k} x^{i} z^{k}, \\
& \varphi_{2}(x, z)=\sum_{i=1}^{\infty} \sum_{k=1}^{\infty} \pi_{2, i, k} x^{i} z^{k}
\end{aligned}
$$


and the auxiliary functions

$$
\begin{aligned}
& \varphi_{1, i}(z)=\sum_{k=0}^{\infty} \pi_{1, i, k} z^{k}, \\
& \varphi_{2, i}(z)=\sum_{k=1}^{\infty} \pi_{2, i, k} z^{k} .
\end{aligned}
$$

Multiplying Eqns. (2) and (3) by $z^{k}$, summing up over $k$ and taking into account Eqn. (1), we get

$$
\begin{aligned}
\varphi_{1, i}(z)= & \bar{a} \varphi_{1, i+1}(z) \\
& +\frac{\bar{a}+a \theta_{1} z(1-z)}{z} \gamma s_{1, i} \varphi_{1,1}(z) \\
& +\frac{\theta_{1} z^{2}+\theta_{2} z+\theta_{3}}{z} a \gamma s_{1, i} \varphi_{1}(1, z) \\
& +\frac{\bar{a}+a z+a \theta_{3}(1-z)}{z} \gamma s_{1, i} \varphi_{2,1}(z) \\
& -\frac{1-z}{z} a\left(1-\theta_{3}\right) \gamma s_{1, i} \pi_{0}, \quad i \geq 1, \\
\varphi_{2, i}(z)= & {\left[\bar{a}+a z+a \theta_{3}(1-z)\right] \varphi_{2, i+1}(z) } \\
& +\left[\bar{a}+a \theta_{1} z(1-z)\right] \bar{\gamma} s_{2, i} \varphi_{1,1}(z) \\
& +\left[\theta_{1} z^{2}+\theta_{2} z+\theta_{3}\right] a \bar{\gamma} s_{2, i} \varphi_{1}(1, z) \\
& +\left[\bar{a}+a z+a \theta_{3}(1-z)\right] \bar{\gamma} s_{2, i} \varphi_{2,1}(z) \\
& -a(1-z)\left(1-\theta_{3}\right) \bar{\gamma} s_{2, i} \pi_{0}, \quad i \geq 1 .
\end{aligned}
$$

Next, multiplying Eqns. (4) and (5) by $x^{i}$ and summing up over $i$, we obtain

$$
\begin{aligned}
\frac{x-\bar{a}}{x} \varphi_{1}(x, z) & {\left[\frac{\bar{a}+a \theta_{1} z(1-z)}{z} \gamma S_{1}(x)-\bar{a}\right] \varphi_{1,1}(z) } \\
= & \frac{\theta_{1} z^{2}+\theta_{2} z+\theta_{3}}{z} a \gamma S_{1}(x) \varphi_{1}(1, z) \\
+ & \frac{\bar{a}+a z+a \theta_{3}(1-z)}{z} \gamma S_{1}(x) \varphi_{2,1}(z) \\
& -\frac{1-z}{z} a\left(1-\theta_{3}\right) \gamma S_{1}(x) \pi_{0}, \\
\frac{x-[a}{a} & \left.+a z+a \theta_{3}(1-z)\right] \\
x & {\left[\bar{a}+a \theta_{1} z(1-z)\right] \bar{\gamma} S_{2}(x) \varphi_{1,1}(z) } \\
& +\left[\theta_{1} z^{2}+\theta_{2} z+\theta_{3}\right] a \bar{\gamma} S_{2}(x) \varphi_{1}(1, z) \\
& +\left\{\left[\bar{a}+a z+a \theta_{3}(1-z)\right] \bar{\gamma} S_{2}(x)\right. \\
& \left.-\left[\bar{a}+a z+a \theta_{3}(1-z)\right]\right\} \varphi_{2,1}(z) \\
& -a(1-z)\left(1-\theta_{3}\right) \bar{\gamma} S_{2}(x) \pi_{0} .
\end{aligned}
$$

Letting $x=1$ in Eqn. (6) gives

$$
\begin{aligned}
a \varphi_{1}(1, z)= & \frac{1}{z-\gamma\left[\theta_{1} z^{2}+\theta_{2} z+\theta_{3}\right]} \\
& \times\left[\left[\left(\bar{a}+a \theta_{1} z(1-z)\right) \gamma-\bar{a} z\right] \varphi_{1,1}(z)\right. \\
& +\left[\bar{a}+a z+a \theta_{3}(1-z)\right] \gamma \varphi_{2,1}(z) \\
& \left.-(1-z) a\left(1-\theta_{3}\right) \gamma \pi_{0}\right]
\end{aligned}
$$

Inserting the above equation into (6) and (7) yields

$$
\begin{aligned}
& \frac{x-\bar{a}}{x} \varphi_{1}(x, z) \\
& =\frac{1}{z-\gamma\left[\theta_{1} z^{2}+\theta_{2} z+\theta_{3}\right]} \\
& \quad \times\left[\left[\theta_{1} z+\bar{a}\left(1-\theta_{3}\right)\right](1-z) \gamma S_{1}(x)\right. \\
& \left.\quad-\bar{a}\left(z-\gamma\left[\theta_{1} z^{2}+\theta_{2} z+\theta_{3}\right]\right)\right] \varphi_{1,1}(z) \\
& \quad+\bar{a}+a z+a \theta_{3}(1-z) \gamma S_{1}(x) \varphi_{2,1}(z) \\
& \left.\quad-(1-z)\left(1-\theta_{3}\right) a \gamma S_{1}(x) \pi_{0}\right], \\
& \frac{x-\left[\bar{a}+a z+a \theta_{3}(1-z)\right]}{\quad x} \varphi_{2}(x, z) \\
& =\frac{1}{z-\gamma\left[\theta_{1} z^{2}+\theta_{2} z+\theta_{3}\right]} \\
& \quad \times\left[\left(\theta_{1} z+\bar{a}\left(1-\theta_{3}\right)\right)(1-z) z \bar{\gamma} S_{2}(x) \varphi_{1,1}(z)\right. \\
& \quad+\left[\bar{a}+a z+a \theta_{3}(1-z)\right]\left[z \bar{\gamma} S_{2}(x)-z\right. \\
& \left.\quad+\gamma\left[\theta_{1} z^{2}+\theta_{2} z+\theta_{3}\right]\right] \varphi_{2,1}(z) \\
& \left.\quad-a z(1-z)\left(1-\theta_{3}\right) \bar{\gamma} S_{2}(x) \pi_{0}\right] .
\end{aligned}
$$

Letting $x=\bar{a}$ and $x=\bar{a}+a z+a \theta_{3}(1-z)$ in (8) and (9), respectively, we have

$$
\varphi_{1,1}(z)=\frac{(1-z)\left(1-\theta_{3}\right) a \gamma S_{1}(\bar{a})}{D(z)} \pi_{0},
$$

$$
\begin{aligned}
\varphi_{2,1}(z)= & \frac{1}{\left[\bar{a}+a z+a \theta_{3}(1-z)\right] \gamma S_{1}(\bar{a}) D(z)} \\
& \times\left[a \bar{a} \bar{\gamma} z(1-z)\left(1-\theta_{3}\right)\right. \\
& \left.\times S_{2}\left(\bar{a}+a z+a \theta_{3}(1-z)\right)\right] \pi_{0},
\end{aligned}
$$

where

$$
\begin{aligned}
D(z)= & {\left[\theta_{1} z+\bar{a}\left(1-\theta_{3}\right)\right](1-z) \gamma S_{1}(\bar{a}) } \\
& +\bar{a} \bar{\gamma} z S_{2}\left(\bar{a}+a z+a \theta_{3}(1-z)\right) \\
& -\bar{a}\left[z-\gamma\left(\theta_{1} z^{2}+\theta_{2} z+\theta_{3}\right)\right] .
\end{aligned}
$$

Substituting the above relations into (8) and (9), we have the following generating functions:

$$
\begin{aligned}
\varphi_{1}(x, z)= & \frac{S_{1}(x)-S_{1}(\bar{a})}{x-\bar{a}} \frac{a \bar{a} \gamma x(1-z)\left(1-\theta_{3}\right)}{D(z)} \pi_{0}, \\
\varphi_{2}(x, z)= & \frac{S_{2}(x)-S_{2}\left(\bar{a}+a z+a \theta_{3}(1-z)\right)}{x-\left(\bar{a}+a z+a \theta_{3}(1-z)\right)} \\
& \times \frac{a \bar{a} \bar{\gamma} x z(1-z)\left(1-\theta_{3}\right)}{D(z)} \pi_{0} .
\end{aligned}
$$

Using the normalization condition $\pi_{0}+\varphi_{1}(1,1)+$ $\varphi_{2}(1,1)=1$, we can find the unknown constant $\pi_{0}$ :

$$
\begin{aligned}
\pi_{0}= & \frac{1}{\gamma\left[\theta_{1} S_{1}(\bar{a})+\bar{a}\left(1-\theta_{1}\right)\right]} \\
& \times\left[\left[\theta_{1}+\bar{a}\left(1-\theta_{3}\right)\right] \gamma S_{1}(\bar{a})\right. \\
& \left.-a \bar{a} \bar{\gamma}\left(1-\theta_{3}\right) \beta_{2,1}-\bar{a} \gamma\left(\theta_{1}-\theta_{3}\right)\right] .
\end{aligned}
$$


Since $\pi_{0}>0$, we obtain that a necessary condition for the system stability is

$$
\begin{aligned}
{\left[\theta_{1}+\bar{a}\left(1-\theta_{3}\right)\right] \gamma S_{1}(\bar{a})-a \bar{a} \bar{\gamma} } & \left(1-\theta_{3}\right) S_{2}^{\prime}(1) \\
& -\bar{a} \gamma\left(\theta_{1}-\theta_{3}\right)>0 .
\end{aligned}
$$

Let us note that the stability condition can be also written as $\rho_{1}+\rho_{2}<1$.

Applying Foster's theorem, it is not difficult to prove that the above condition is also sufficient for system stability. In what follows, when we consider stationary distributions of any system's characteristics, it will always be supposed that the stability condition is fulfilled.

The above results can be summarized in the following theorem.

Theorem 1. If $\rho_{1}+\rho_{2}<1$, the stationary distribution of the Markov chain $\left\{\left(C_{m}, \xi_{1, m}, \xi_{2, m}, N_{m}\right), m \in \mathbb{N}\right\}$ has the following generating functions:

$$
\begin{aligned}
\varphi_{1}(x, z)= & \frac{S_{1}(x)-S_{1}(\bar{a})}{x-\bar{a}} \frac{a \bar{a} \gamma x(1-z)\left(1-\theta_{3}\right)}{D(z)} \pi_{0}, \\
\varphi_{2}(x, z)= & \frac{S_{2}(x)-S_{2}\left(\bar{a}+a z+a \theta_{3}(1-z)\right)}{x-\left(\bar{a}+a z+a \theta_{3}(1-z)\right)} \\
& \times \frac{a \bar{a} \bar{\gamma} x z(1-z)\left(1-\theta_{3}\right)}{D(z)} \pi_{0},
\end{aligned}
$$

where

$$
\begin{aligned}
\pi_{0}= & \frac{1}{\gamma\left[\theta_{1} S_{1}(\bar{a})+\bar{a}\left(1-\theta_{1}\right)\right]} \\
& \times\left[\left[\theta_{1}+\bar{a}\left(1-\theta_{3}\right)\right] \gamma S_{1}(\bar{a})\right. \\
& \left.-a \bar{a} \bar{\gamma}\left(1-\theta_{3}\right) \beta_{2,1}-\bar{a} \gamma\left(\theta_{1}-\theta_{3}\right)\right] .
\end{aligned}
$$

\section{Corollary 1.}

1. The marginal generating function of the number of customers in the queue when the server is busy is given by

$$
\varphi_{1}(1, z)=\frac{\left(1-S_{1}(\bar{a})\right) \bar{a} \gamma\left(1-\theta_{3}\right)(1-z)}{D(z)} \pi_{0} .
$$

2. The marginal generating function of the number of customers in the queue when the server is down is given by

$\varphi_{2}(1, z)=\frac{\left[1-S_{1}\left(\bar{a}+a z+a \theta_{3}(1-z)\right)\right] \bar{a} \bar{\gamma} z}{D(z)} \pi_{0}$.

3. The probability generating function of the queue size (i.e., of the variable $N$ ) is given by

$$
\begin{aligned}
\Psi(z) & =\pi_{0}+\varphi_{1}(1, z)+\varphi_{2}(1, z) \\
& =\frac{(1-z)\left[\theta_{1} z S_{1}(\bar{a})+\bar{a}\left(1-\theta_{1} z\right)\right]}{D(z)} \gamma \pi_{0} .
\end{aligned}
$$

4. The probability generating function of the system size (i.e., of the variable L) is given by

$$
\begin{aligned}
\Phi(z)= & \pi_{0}+z \varphi_{1}(1, z)+\varphi_{2}(1, z) \\
= & \frac{1}{D(z)}(1-z)\left[\theta_{1} z S_{1}(\bar{a})+\bar{a}\left(1-\theta_{1} z\right)\right. \\
& \left.-\bar{a}\left(1-\theta_{3}\right)(1-z)\left(1-S_{1}(\bar{a})\right)\right] \gamma \pi_{0} .
\end{aligned}
$$

Differentiating the above GFs at the point $z=1$, we can obtain the stationary characteristic of the mean queue and the mean system size.

\section{Stationary characteristics}

In this section we present some performance measures for the system at the stationary regime.

4.1. Probability that the sever is respectively idle, busy and down.

$$
\begin{aligned}
\pi_{0} & =1-\rho_{1}-\rho_{2}, \\
\varphi_{1}(1,1) & =\rho_{1}, \\
\varphi_{2}(1,1) & =\rho_{2} .
\end{aligned}
$$

\subsection{Mean queue size.}

$$
\begin{aligned}
E[N]= & \Psi^{\prime}(1) \\
= & {\left[2 \theta_{1} \gamma\left(\bar{a}-S_{1}(\bar{a})\right)\left(1-S_{1}(\bar{a})\right)+2 a \bar{a} \bar{\gamma} \beta_{2,1}\right.} \\
& \left.+\left[\bar{a}-\theta_{1}\left(\bar{a}-S_{1}(\bar{a})\right)\right] a^{2} \bar{\gamma}\left(1-\theta_{3}\right) \beta_{2,2}\right] \bar{a}\left(1-\theta_{3}\right) \\
& \times\left[2 \left[\gamma S_{1}(\bar{a})\left(\theta_{1}+\bar{a}\left(1-\theta_{3}\right)\right)-a \bar{a}\left(1-\theta_{3}\right) \bar{\gamma} \beta_{2,1}\right.\right. \\
& \left.\left.-\bar{a} \gamma\left(\theta_{1}-\theta_{3}\right)\right]\left[\theta_{1} S_{1}(\bar{a})+\bar{a}\left(1-\theta_{1}\right)\right]\right]^{-1} .
\end{aligned}
$$

\subsection{Mean system size.}

$$
E[L]=E[N]+\bar{\nu},
$$

where $\bar{\nu}$ is the mean number of customers in the server.

Let us observe that the GF of the number of customers in the server is given by

$$
\nu(z)=1-\frac{\bar{a}\left(1-\theta_{3}\right)(1-z)\left(1-S_{1}(\bar{a})\right)}{\theta_{1} z S_{1}(\bar{a})+\bar{a}\left(1-\theta_{1} z\right)},
$$

and

$$
\bar{\nu}=\nu^{\prime}(1)=\frac{\bar{a}\left(1-\theta_{3}\right)\left(1-S_{1}(\bar{a})\right)}{\theta_{1} S_{1}(\bar{a})+\bar{a}\left(1-\theta_{1}\right)} .
$$

Remark 1. It can be observed that $\Phi(z)=\nu(z) \Psi(z)$, and, as a consequence, we find the formula, for $n \geq 1$,

$$
\begin{aligned}
\Phi^{(n)}(1)= & \sum_{m=0}^{n}\left(\begin{array}{c}
n \\
m
\end{array}\right) \frac{m !\left[\theta_{1}\left(\bar{a}-S_{1}(\bar{a})\right)\right]^{m-1}}{\left[\theta_{1} S_{1}(\bar{a})+\bar{a}\left(1-\theta_{1}\right)\right]^{m}} . \\
& \times \bar{a}\left(1-\theta_{3}\right)\left(1-S_{1}(\bar{a})\right) \Psi^{(n-m)}(1) .
\end{aligned}
$$


Special cases. As mentioned in the abstract, the versatility of the model, that is, its capacity for describing different situations and disciplines, due to the variety of parameters involved, makes interesting to point out the special cases more relevant.

Case 1. If in our system we consider $\theta_{2}=0$, we get

$$
\begin{aligned}
\Phi_{\theta_{2}=0}= & (1-z)\left[\theta_{1}(\bar{a}+a z) S_{1}(\bar{a})+\bar{a}\left(1-\theta_{1}\right)\right] \\
& \times\left[\theta_{1}(z+\bar{a})(1-z) \gamma S_{1}(\bar{a})\right. \\
& +\bar{a} \bar{\gamma} z S_{2}\left(1-a \theta_{1}(1-z)\right) \\
& \left.-\bar{a}\left[z-\gamma\left(1-\theta_{1}\left(1-z^{2}\right)\right)\right]\right]^{-1} \gamma \pi_{0},
\end{aligned}
$$

where

$$
\pi_{0}=\frac{\theta_{1}(1+\bar{a}) \gamma S_{1}(\bar{a})-a \bar{a} \bar{\gamma} \theta_{1} \beta_{21}+\bar{a} \gamma\left(1-2 \theta_{1}\right)}{\gamma\left[\theta_{1} S_{1}(\bar{a})+\bar{a}\left(1-\theta_{1}\right)\right]},
$$

which is the GF of the number of customers in the $G e o / G / 1 / \infty$ queueing system with negative customers, the LCFS discipline and starting failures.

Case 2. Considering the case where $\theta_{1}=1$, we have

$$
\begin{aligned}
\Phi_{\theta_{1}=1}= & (1-z)(\bar{a}+a z) S_{1}(\bar{a}) \\
& \times\left[(z+\bar{a})(1-z) \gamma S_{1}(\bar{a})\right. \\
& +\bar{a} \bar{\gamma} z S_{2}(\bar{a}+a z) \\
& -\bar{a} z(1-\gamma z)]^{-1} \gamma \pi_{0},
\end{aligned}
$$

where

$$
\pi_{0}=\frac{(1+\bar{a}) \gamma S_{1}(\bar{a})-a \bar{a} \bar{\gamma} \beta_{21}-\bar{a} \gamma}{\gamma S_{1}(\bar{a})}
$$

which is the GF of the number of customers in the $G e o / G / 1 / \infty$ queueing system with repairs and the LCFS discipline.

Case 3. Let us note that if in our system we do not consider expulsions and starting failures, that is, $\theta_{1}=\gamma=$ 1 , we have

$$
\Phi^{*}(z)=\frac{(\bar{a}+a z) S_{1}(\bar{a})}{\bar{a} S_{1}(\bar{a})-z\left[\bar{a}-S_{1}(\bar{a})\right]} \pi_{0}^{*},
$$

where

$$
\pi_{0}^{*}=\frac{\bar{a} S_{1}(\bar{a})-\left[\bar{a}-S_{1}(\bar{a})\right]}{S_{1}(\bar{a})},
$$

which is the GF of the number of customers in the $G e o / G / 1 / \infty$ queueing system with the LCFS discipline.

In this case, if we consider geometric services with parameter $b$, the corresponding GF is $S(z)=b z /(1-\bar{b} z)$ and the GF of the number of customers in the system is

$$
\Phi(z)=\frac{\bar{a}+a z}{\bar{a}(1-\delta z)} p_{0},
$$

where $p_{0}=1-a / b$ and $\delta=a \bar{b} /(\bar{a} b)$, which is the GF of the standard Geo/Geo/1/ $\infty$ with the LCFS and also the FCFS discipline. This result is far from surprising due to the memoryless property of the geometrical distribution.

Case 4. If in our system we consider $\gamma=1$ and $\theta_{2}=0$, we have

$$
\Phi^{*}(z)=\frac{\theta_{1} S_{1}(\bar{a})(\bar{a}+a z)+\bar{a} \theta_{3}}{\theta_{1}(\bar{a}+z) S_{1}(\bar{a})+\bar{a}\left[1-\theta_{1}(1+z)\right]} \pi_{0}^{*},
$$

where

$$
\pi_{0}^{*}=\frac{\theta_{1}(\bar{a}+1) S_{1}(\bar{a})+\bar{a}\left(1-2 \theta_{1}\right)}{\theta_{1} S_{1}(\bar{a})+\bar{a} \theta_{3}} .
$$

which is the GF of the number of customers in the $G e o / G / 1 / \infty$ queueing system with negative customers and the LCFS discipline.

\section{Busy period}

A busy period is defined to begin with the arrival of a customer to an empty system and to end when the system next becomes empty. In this section we will study the busy period of an auxiliary system, which will be useful to study the customer delay in the original system.

This auxiliary system differs from the original by the fact that the customer who enters the system goes directly to the server, interrupting the service of the customer who is currently being served, if any. Therefore, we do not consider the possibility that any customer enters the system during the repair times. We will denote by $h_{k}$, $k \geq 0$, the probability that the busy period lasts $k$ slots. Then we have

$$
\begin{aligned}
h_{0}= & 0 \\
h_{k}= & \gamma\left[\bar{a}^{k-1} s_{1, k}\left(\bar{a}+a \theta_{3}\right)\right. \\
& +\sum_{i=1}^{k} \bar{a}^{i-1} s_{1, i} a\left(1-\theta_{3}\right) h_{k-i} \\
& +\sum_{i=1}^{k} \bar{a}^{i-1} S_{1, i+1} a \theta_{1} \sum_{j=0}^{k-i} h_{j} h_{k-i-j} \\
& \left.+\sum_{i=1}^{k} \bar{a}^{i-1} S_{1, i+1} a \theta_{2} h_{k-i}+\bar{a}^{k-1} S_{1, k+1} a \theta_{3}\right] \\
& +\bar{\gamma}\left[\sum_{i=1}^{k} s_{2, i} a\left(1-\theta_{3}\right) \sum_{j=0}^{k-i} h_{j} h_{k-i-j}\right. \\
& \left.+\sum_{i=1}^{k} s_{2, i}\left(\bar{a}+a \theta_{3}\right) h_{k-i}\right], \quad k \geq 1 .
\end{aligned}
$$

The above formulae can be used recursively in $k$ to numerically calculate the distribution $\left\{h_{k}, k \geq 0\right\}$. Nevertheless, with the aim of calculating the moments of 
the distribution, we will work with its GF $h(x)$ that has the following form:

$$
\begin{aligned}
h(x)= & \sum_{k=0}^{\infty} x^{k} h_{k} \\
= & \gamma\left[\frac{\bar{a}+a \theta_{3}}{\bar{a}} S_{1}(\bar{a} x)+\frac{a\left(1-\theta_{3}\right)}{\bar{a}} S_{1}(\bar{a} x) h(x)\right. \\
& +\frac{a \theta_{1}}{\bar{a}} \frac{\bar{a} x-S_{1}(\bar{a} x)}{1-\bar{a} x} h^{2}(x) \\
& \left.+\frac{a \theta_{2}}{\bar{a}} \frac{\bar{a} x-S_{1}(\bar{a} x)}{1-\bar{a} x} h(x)+\frac{a \theta_{3}}{\bar{a}} \frac{\bar{a} x-S_{1}(\bar{a} x)}{(1-\bar{a} x)}\right] \\
& +\bar{\gamma}\left[a\left(1-\theta_{3}\right) S_{2}(x) h^{2}(x)\right. \\
& \left.+\left(\bar{a}+a \theta_{3}\right) S_{2}(x) h(x)\right] .
\end{aligned}
$$

The above formula can be expressed in the form

$$
\begin{aligned}
& {\left[a \theta_{1} \gamma\left[\bar{a} x-S_{1}(\bar{a} x)\right]\right.} \\
& \left.\quad+a \bar{a}\left(1-\theta_{3}\right) \bar{\gamma}(1-\bar{a} x) S_{2}(x)\right] h^{2}(x) \\
& \quad-\left[\bar{a}(1-\bar{a} x)-a\left(1-\theta_{3}\right) \gamma(1-\bar{a} x) S_{1}(\bar{a} x)\right. \\
& \quad-a \theta_{2} \gamma\left[\bar{a} x-S_{1}(\bar{a} x)\right] \\
& \left.\quad-\bar{a}\left(\bar{a}+a \theta_{3}\right) \bar{\gamma}(1-\bar{a} x) S_{2}(x)\right] h(x) \\
& \quad+\gamma\left[\left(\bar{a}+a \theta_{3}\right)(1-\bar{a} x) S_{1}(\bar{a} x)\right. \\
& \left.\quad+a \theta_{3}\left[\bar{a} x-S_{1}(\bar{a} x)\right]\right]=0 .
\end{aligned}
$$

Therefore, the GF $h=h(x)$ satisfies the quadratic equation

$$
f(h)=0,
$$

where

$$
\begin{aligned}
f(h)= & {\left[\gamma a \theta_{1}\left(\bar{a} x-S_{1}(\bar{a} x)\right)\right.} \\
& \left.+\bar{\gamma} a\left(1-\theta_{3}\right) \bar{a}(1-\bar{a} x) S_{2}(x)\right] h^{2} \\
& -\left[\bar{a}(1-\bar{a} x)-\gamma(1-\bar{a} x) a\left(1-\theta_{3}\right) S_{1}(\bar{a} x)\right. \\
& -\gamma a \theta_{2}\left(\bar{a} x-S_{1}(\bar{a} x)\right) \\
& \left.+\bar{\gamma} \bar{a}(1-\bar{a} x)\left(\bar{a}+a \theta_{3}\right) S_{2}(x)\right] h \\
& +\gamma\left[(1-\bar{a} x)\left(\bar{a}+a \theta_{3}\right) S_{1}(\bar{a} x)\right. \\
& \left.+\left(\bar{a} x-S_{1}(\bar{a} x)\right) a \theta_{3}\right]
\end{aligned}
$$

Let us note that for any fixed $x_{0} \in(0,1)$ we have

$$
\begin{aligned}
\gamma a \theta_{1}\left(\bar{a} x_{0}-\right. & \left.S_{1}\left(\bar{a} x_{0}\right)\right) \\
& +\bar{\gamma} a\left(1-\theta_{3}\right) \bar{a}\left(1-\bar{a} x_{0}\right) S_{2}\left(x_{0}\right)>0, \\
f(0)= & \gamma\left[\left(1-\bar{a} x_{0}\right)\left(\bar{a}+a \theta_{3}\right) S_{1}\left(\bar{a} x_{0}\right)\right. \\
& \left.+\left(\bar{a} x_{0}-S_{1}\left(\bar{a} x_{0}\right)\right) a \theta_{3}\right]>0 \\
f(1)= & \bar{a}\left(x_{0}-1\right)\left[1-\gamma S_{1}\left(\bar{a} x_{0}\right)\right] \\
& +\bar{a} \bar{\gamma}\left[\left(1-\bar{a} x_{0}\right) S_{2}\left(x_{0}\right)-a x_{0}\right]<0 .
\end{aligned}
$$

The above relations show that for any $x \in(0,1)$ Eqn. (12) has two solutions, $h(x)$ and $h^{*}(x)$, satisfying the inequalities $0<h(x)<1<h^{*}(x)$ and given by

$$
\begin{aligned}
h(x)= & \frac{1}{\mathcal{D}(x)} \\
& \times\left[\bar{a}(1-\bar{a} x)-\gamma(1-\bar{a} x) a\left(1-\theta_{3}\right) S_{1}(\bar{a} x)\right. \\
& -\gamma a \theta_{2}\left(\bar{a} x-S_{1}(\bar{a} x)\right) \\
& \left.+\bar{\gamma} \bar{a}(1-\bar{a} x)\left(\bar{a}+a \theta_{3}\right) S_{2}(x)-\mathcal{D}(x) u(x)\right], \\
h^{*}(x)= & \frac{1}{\mathcal{D}(x)} \\
& \times\left[\bar{a}(1-\bar{a} x)-\gamma(1-\bar{a} x) a\left(1-\theta_{3}\right) S_{1}(\bar{a} x)\right. \\
& -\gamma a \theta_{2}\left(\bar{a} x-S_{1}(\bar{a} x)\right) \\
& \left.+\bar{\gamma} \bar{a}(1-\bar{a} x)\left(\bar{a}+a \theta_{3}\right) S_{2}(x)+\mathcal{D}(x) u(x)\right],
\end{aligned}
$$

where

$$
\begin{aligned}
\mathcal{D}(x)= & 2\left[a \theta_{1} \gamma\left(\bar{a} x-S_{1}(\bar{a} x)\right)\right. \\
& \left.+a \bar{a} \bar{\gamma}\left(1-\theta_{3}\right)(1-\bar{a} x) S_{2}(x)\right], \\
u(x)= & \left(\left[\bar{a}(1-\bar{a} x)-\gamma(1-\bar{a} x) a\left(1-\theta_{3}\right) S_{1}(\bar{a} x)\right.\right. \\
& -\gamma a \theta_{2}\left(\bar{a} x-S_{1}(\bar{a} x)\right) \\
& \left.+\bar{\gamma} \bar{a}(1-\bar{a} x)\left(\bar{a}+a \theta_{3}\right) S_{2}(x)\right]^{2} \\
& -4\left[\gamma a \theta_{1}\left(\bar{a} x-S_{1}(\bar{a} x)\right)\right. \\
& \left.+\bar{\gamma} a\left(1-\theta_{3}\right) \bar{a}(1-\bar{a} x) S_{2}(x)\right] \\
& \times \gamma\left[(1-\bar{a} x)\left(\bar{a}+a \theta_{3}\right) S_{1}(\bar{a} x)\right. \\
& \left.\left.+\left(\bar{a} x-S_{1}(\bar{a} x)\right) a \theta_{3}\right]\right)^{1 / 2} \\
& \times\left(2 \gamma a \theta_{1}\left(\bar{a} x-S_{1}(\bar{a} x)\right)\right. \\
& \left.+\bar{\gamma} a\left(1-\theta_{3}\right) \bar{a}(1-\bar{a} x) S_{2}(x)\right)^{-1} .
\end{aligned}
$$

For $x=1$ we have $f(1)=0$, which implies that at least one of the solutions takes the value 1 for $x=1$.

The GF of the busy period is defined by the first (minimal) solution $h(x)$. In order to check that $h(1)=1$, we will show that, if the stability condition is fulfilled, $h^{*}(1)>1$, that is,

$$
\frac{B+\sqrt{B^{2}-4 A C}}{2 A}>1,
$$

where

$$
\begin{aligned}
A= & a \theta_{1} \gamma\left(\bar{a}-S_{1}(\bar{a})\right)+a^{2} \bar{a}\left(1-\theta_{3}\right) \bar{\gamma} \\
B= & a \bar{a}-a^{2}\left(1-\theta_{3}\right) \gamma S_{1}(\bar{a})-a \theta_{2} \gamma\left(\bar{a}-S_{1}(\bar{a})\right) \\
& -a \bar{a}\left(\bar{a}+a \theta_{3}\right) \bar{\gamma} \\
C= & a \gamma\left[\left(\bar{a}+a \theta_{3}\right) S_{1}(\bar{a})+\theta_{3}\left(\bar{a}-S_{1}(\bar{a})\right)\right] .
\end{aligned}
$$

The former inequality can be written as

$$
\begin{aligned}
\sqrt{B^{2}-4 A C}> & 2 A-B \\
= & a\left[a \bar{a} \bar{\gamma}\left(1-\theta_{3}\right)+\bar{a} \gamma\left(\theta_{1}-\theta_{3}\right)\right. \\
& \left.-\left[\theta_{1}+\bar{a}\left(1-\theta_{3}\right)\right] \gamma S_{1}(\bar{a})\right],
\end{aligned}
$$

but the right-hand side of the above inequality is negative because of the stability condition and, in consequence $h^{*}(1)>1$. Therefore the busy period is $h(x)$. 
Let us note that the value of $h^{*}(1)$ can be found as

$$
h^{*}(1)=\frac{\bar{a} \gamma\left[\left(1-\theta_{3}\right) S_{1}(\bar{a})+\theta_{3}\right]}{\theta_{1} \gamma\left(\bar{a}-S_{1}(\bar{a})\right)+a \bar{a} \bar{\gamma}\left(1-\theta_{3}\right)},
$$

which is greater than 1 if the stability condition is satisfied.

The mean length of a busy period is given by

$$
\begin{aligned}
\bar{h}= & h^{\prime}(1) \\
= & {\left[\gamma\left(1-S_{1}(\bar{a})\right)+a \bar{\gamma} S_{2}^{\prime}(1)\right] \frac{\bar{a}}{a} } \\
& \times\left[\left[\theta_{1}+\bar{a}\left(1-\theta_{3}\right)\right] \gamma S_{1}(\bar{a})-a \bar{a} \bar{\gamma}\left(1-\theta_{3}\right)\right. \\
& \left.-\bar{a} \gamma\left(\theta_{1}-\theta_{3}\right)\right]^{-1} .
\end{aligned}
$$

In order to find the generating function of the waiting time of a customer in the queue, we need to consider the busy period, which begins when the server is down and the remaining repair time is $i$ slots and there is one customer in the queue (the one with the repair began).

Let us denote by $h(i, l)$ the probability that this busy period lasts $l$ slots, $l \geq i+1$. Then

$$
h(i, l)=\left(\bar{a}+a \theta_{3}\right) h_{l-i}+a\left(\theta_{1}+\theta_{2}\right) \sum_{j=0}^{l-i} h_{j} h_{l-i-j} .
$$

The corresponding GF is given by

$$
\begin{aligned}
h(x, i) & =\sum_{l=i+1}^{\infty} h(i, l) x^{l} \\
& =\left[\left(\bar{a}+a \theta_{3}\right) h(x)+a\left(\theta_{1}+\theta_{2}\right) h^{2}(x)\right] x^{i} .
\end{aligned}
$$

\section{Sojourn times}

6.1. Sojourn time of a customer in the server. In this section we are going to find the distribution of the time that a customer spends in the server. We will denote by $b_{k}$ the probability that the sojourn time of a customer in the server lasts exactly $k$ slots. The distribution $\left\{b_{k} ; k \geq 0\right\}$ is given by

$$
\begin{aligned}
b_{0}= & 0 \\
b_{k}= & \bar{a}^{k-1} s_{1, k}+\sum_{i=1}^{k} \bar{a}^{i-1} a \theta_{1} S_{1, i+1} b_{k-i} \\
& +a\left(\theta_{2}+\theta_{3}\right) \bar{a}^{k-1} S_{1, k+1} .
\end{aligned}
$$

The corresponding GF $b(x)=\sum_{k=0}^{\infty} x^{k} b_{k}$ is given by

$$
b(x)=\frac{(1-\bar{a} x) S_{1}(\bar{a} x)+a\left(\theta_{2}+\theta_{3}\right)\left(\bar{a} x-S_{1}(\bar{a} x)\right)}{\bar{a}(1-\bar{a} x)-a \theta_{1}\left(\bar{a} x-S_{1}(\bar{a} x)\right)} .
$$

The mean sojourn time of a customer in the server is

$$
\bar{b}=b^{\prime}(1)=\frac{1-S_{1}(\bar{a})}{\bar{a}\left(1-\theta_{1}\right)+\theta_{1} S_{1}(\bar{a})} \frac{\bar{a}}{a} .
$$

Let us note that

$$
\bar{\nu}=a\left(1-\theta_{3}\right) \bar{b},
$$

as Little's principle establishes.
6.2. Sojourn time of a customer in the system. Firstly, we will find the distribution of the period of time that a customer spends in the system from the beginning of its service till the moment of its departure. Let $g_{k}$ be the probability that this period of time lasts exactly $k$ slots. Then we have

$$
\begin{aligned}
g_{0}= & 0 \\
g_{k}= & \gamma\left[\bar{a}^{k-1} s_{1, k}+a\left(\theta_{2}+\theta_{3}\right) \bar{a}^{k-1} S_{1, k+1}\right. \\
& \left.+\sum_{i=1}^{k} \bar{a}^{i-1} S_{1, i+1} a \theta_{1} \sum_{j=0}^{k-i} h_{j} g_{k-i-j}\right] \\
& +\bar{\gamma}\left[\sum_{i=1}^{k} s_{2, i}\left(\bar{a}+a \theta_{3}\right) g_{k-i}\right. \\
& \left.+\sum_{i=1}^{k} s_{2, i} a\left(1-\theta_{3}\right) \sum_{j=0}^{k-i} h_{j} g_{k-i-j}\right],
\end{aligned}
$$

and the corresponding GF is

$$
\begin{aligned}
g(x)= & \sum_{k=0}^{\infty} x^{k} g_{k} \\
= & \gamma\left[\frac{S_{1}(\bar{a} x)}{\bar{a}}+a\left(\theta_{2}+\theta_{3}\right) \frac{\bar{a} x-S_{1}(\bar{a} x)}{\bar{a}(1-\bar{a} x)}\right. \\
& \left.+\frac{a \theta_{1}}{\bar{a}} \frac{\bar{a} x-S_{1}(\bar{a} x)}{1-\bar{a} x} h(x) g(x)\right] \\
& +\bar{\gamma}\left[S_{2}(x) g(x)\left(\bar{a}+a \theta_{3}\right)\right. \\
& \left.+a\left(1-\theta_{3}\right) S_{2}(x) h(x) g(x)\right]
\end{aligned}
$$

that is,

$$
\begin{aligned}
g(x)= & {\left[(1-\bar{a} x) S_{1}(\bar{a} x)\right.} \\
& \left.+a\left(\theta_{2}+\theta_{3}\right)\left(\bar{a} x-S_{1}(\bar{a} x)\right)\right] \gamma \\
& \times\left[\bar{a}(1-\bar{a} x)-\left[a \theta_{1} \gamma\left[\bar{a} x-S_{1}(\bar{a} x)\right]\right.\right. \\
& \left.+a \bar{a} \bar{\gamma}\left(1-\theta_{3}\right)(1-\bar{a} x)\right] h(x) \\
& \left.-\bar{a} \bar{\gamma}\left(\bar{a}+a \theta_{3}\right)(1-\bar{a} x) S_{2}(x)\right]^{-1} .
\end{aligned}
$$

The mean length of the period of time that a customer spends from the beginning of its service till the moment of leaving the system is given by

$$
\begin{aligned}
\bar{g}= & g^{\prime}(1) \\
= & \frac{1}{a \gamma\left[\bar{a}\left(1-\theta_{1}\right)+\theta_{1} S_{1}(\bar{a})\right]} \bar{a} \gamma\left(1-S_{1}(\bar{a})\right) \\
& +a \bar{h}\left[\gamma \theta_{1}\left(\bar{a}-S_{1}(\bar{a})\right)+a \bar{a} \bar{\gamma}\left(1-\theta_{3}\right)\right]+a \bar{a} \bar{\gamma} S_{2}^{\prime}(1) .
\end{aligned}
$$

We are going to study the period of time that a customer spends in the queue from the moment of his/her arrival to the system till the moment of the beginning of the service. Let us note that the period of time that a customer spends in the system from the moment of the beginning of the service till the moment of the departure has been already taken into account in the distribution $\left\{g_{k}, k \geq 0\right\}$. 
In order to find the stationary distribution of the sojourn time of a customer in the queue till the beginning of the service, we note that if an arriving customer finds the system empty or the server busy (all with probability $\left.\pi_{0}+\varphi_{1}(1,1)\right)$ the service will begin immediately and therefore his sojourn time in the queue till the beginning of his/her service will last zero slots. On the other hand, a customer that arrives to the system while the server is down, with the remaining repair time of $i$ slots, and finds $k$ other customers in the queue before him/her (with probability $\pi_{2, i, k}$ ) will spend in the queue till the beginning of his service a period of time formed by the busy period $h(x, i)$ plus the busy periods generated by $k-1$ customers in the queue before him/her, since the first one at the head of the queue has already been taken into account in the busy period $h(x, i)$.

Therefore, the stationary distribution of the sojourn time that a customer spends in the queue until the beginning of his/her service has the following generating function:

$$
\begin{aligned}
w(x)= & \pi_{0}+\varphi_{1}(1,1) \\
& +\left[\left(\bar{a}+a \theta_{3}\right) h(x)+a\left(\theta_{1}+\theta_{2}\right) h^{2}(x)\right] \\
& \times \sum_{i=1}^{\infty} \sum_{k=1}^{\infty} \pi_{2, i, k} x^{i} h^{k-1}(x) \\
= & \pi_{0}+\varphi_{1}(1,1) \\
& +\left[\left(\bar{a}+a \theta_{3}\right)+a\left(\theta_{1}+\theta_{2}\right) h(x)\right] \varphi_{2}(x, h(x)) .
\end{aligned}
$$

The corresponding mean time is

$$
\begin{aligned}
\bar{w}= & w^{\prime}(1) \\
= & \frac{a \bar{a} \bar{\gamma}\left(1-\theta_{3}\right)}{\gamma\left[\theta_{1} S_{1}(\bar{a})+\bar{a}\left(1-\theta_{1}\right)\right]} \\
& \times\left[\frac{2 S_{2}^{\prime}(1)+S_{2}^{\prime \prime}(1)}{2}\left(1+a\left(1-\theta_{3}\right) \bar{h}\right)+E \bar{h} S_{2}^{\prime}(1)\right],
\end{aligned}
$$

where

$$
\begin{aligned}
E= & {\left[2\left(\bar{a}\left(1-\theta_{3}\right) \gamma S_{1}(\bar{a})+\bar{a} \theta_{3} \gamma\right)\right.} \\
& \left.+a^{2} \bar{a}\left(1-\theta_{3}\right)^{2} \bar{\gamma} S_{2}^{\prime \prime}(1)\right] \\
& \times\left[2 \left[\left(\theta_{1}+\bar{a}\left(1-\theta_{3}\right)\right) \gamma S_{1}(\bar{a})\right.\right. \\
& \left.\left.-a \bar{a}\left(1-\theta_{3}\right) \bar{\gamma} S_{2}^{\prime}(1)-\bar{a} \gamma\left(\theta_{1}-\theta_{3}\right)\right]\right]^{-1} .
\end{aligned}
$$

The GF of the sojourn time in the system for a random customer is given by

$$
v(x)=w(x) g(x),
$$

and the mean sojourn time in the system by

$$
\bar{v}=v^{\prime}(1)=\bar{w}+\bar{g} .
$$

\section{Relation to the continuous-time system}

In this section we show how the continuous-time version of our model can be approximated by its discrete-time counterpart. In order to unify the results of both the discrete-time and the corresponding continuous-time models, below we give a succinct proof as to how to get the continuous-time results from those of the discrete-time case. Using appropriate limits, we can get the results of the continuous-time $M / G / 1 / \infty$ queue where customers arrive according to a Poisson process with rate $\lambda$.

The server is subject to starting failures, and the parameters $\gamma, \theta_{1}, \theta_{2}$ and $\theta_{3}$ play the same role as in the discrete-time model. Service and repair times are independent and identically distributed with common distribution $B_{1}(x)$ and $B_{2}(x)$, Laplace-Stieltjes transform $\beta_{1}(s)$ and $\beta_{2}(s)$, and finite means $\mu_{1}^{-1}$ and $\mu_{2}^{-2}$, respectively.

If we suppose that the time axis is divided into equal intervals of length $\Delta$, the above continuous-time system can be approximated by our discrete-time model considering

$$
a=\lambda \Delta
$$

and

$$
s_{j, i}=\int_{(i-1) \Delta}^{i \Delta} \mathrm{d} B_{j}(x), \quad j=1,2, i \geq 1,
$$

where $\Delta$ is sufficiently small so that $a$ is a probability.

The main purpose of this section is to establish that $\lim _{\Delta \rightarrow 0} \Phi(z)$ is the probability generating function of the number of customers in the $M / G / 1 / \infty$ queue described above.

It is not difficult to justify the following equalities using the definition of the Lebesgue integral

$$
\begin{aligned}
& \lim _{\Delta \rightarrow 0} S_{1}(\bar{a}) \\
& =\lim _{\Delta \rightarrow 0} \sum_{i=1}^{\infty}\left[B_{1}(i \Delta)-B_{1}((i-1) \Delta)\right][1-\lambda \Delta]^{i} \\
& =\lim _{\Delta \rightarrow 0} \sum_{i=1}^{\infty}\left[B_{1}(i \Delta)-B_{1}((i-1) \Delta)\right]\left[(1-\lambda \Delta)^{\frac{1}{\Delta}}\right]^{i \Delta} \\
& =\lim _{\Delta \rightarrow 0} \sum_{i=1}^{\infty}\left[B_{1}(i \Delta)-B_{1}((i-1) \Delta)\right]\left[e^{-\lambda+\mathcal{O}(1)}\right]^{i \Delta} \\
& =\int_{0}^{\infty} e^{-\lambda x} \mathrm{~d} B_{1}(x)=\beta_{1}(\lambda) .
\end{aligned}
$$

In a similar way, we obtain

$$
\lim _{\Delta \rightarrow 0} S_{2}\left(\bar{a}+a z+a \theta_{3}(1-z)\right)=\beta_{2}\left(\lambda\left(1-\theta_{3}\right)(1-z)\right) .
$$


We also need the limits

$$
\begin{aligned}
\lim _{\Delta \rightarrow 0} a \beta_{2,1}= & \lim _{\Delta \rightarrow 0} \lambda \Delta \sum_{i=1}^{\infty} i\left[B_{1}(i \Delta)-B_{1}((i-1) \Delta)\right] \\
= & \lim _{\Delta \rightarrow 0} \lambda \sum_{i=1}^{\infty} i \Delta\left[B_{1}(i \Delta)-B_{1}((i-1) \Delta)\right] \\
= & \lambda \int_{0}^{\infty} x \mathrm{~d} B_{2}(x)=\frac{\lambda}{\mu_{2}}, \\
\lim _{\Delta \rightarrow 0} \rho_{1}= & \lim _{\Delta \rightarrow 0} a\left(1-\theta_{3}\right) \bar{b} \\
= & \lim _{\Delta \rightarrow 0} \frac{\left(1-\theta_{3}\right)\left(1-S_{1}(\bar{a})\right)}{\theta_{1} S_{1}(\bar{a})+\bar{a}\left(1-\theta_{1}\right)} \bar{a} \\
= & \lim _{\Delta \rightarrow 0} \frac{\left(1-\theta_{3}\right)\left(1-S_{1}(\bar{a})\right)}{\theta_{1} S_{1}(\bar{a})+(1-\lambda \Delta)\left(1-\theta_{1}\right)} \\
& \times(1-\lambda \Delta) \\
= & \frac{\left(1-\theta_{3}\right)\left(1-\beta_{1}(\lambda)\right)}{\theta_{1} \beta_{1}(\lambda)+1-\theta_{1}},
\end{aligned}
$$

and

$$
\begin{aligned}
\lim _{\Delta \rightarrow 0} \rho_{2} & =\lim _{\Delta \rightarrow 0} \frac{\bar{a} \bar{\gamma}\left(1-\theta_{3}\right) a \beta_{2,1}}{\gamma\left[\theta_{1} S_{1}(\bar{a})+\bar{a}\left(1-\theta_{1}\right)\right]} \\
& =\frac{\left(1-\theta_{3}\right) \bar{\gamma} \lambda \mu_{2}^{-1}}{\gamma\left[\theta_{1} \beta_{1}(\lambda)+1-\theta_{1}\right]} .
\end{aligned}
$$

Thus, the load of the system in the continuous case is given by

$$
\rho_{1}+\rho_{2}=\frac{\left[\gamma\left(1-\beta_{1}(\lambda)\right)+\bar{\gamma} \lambda \mu_{2}^{-1}\right]\left(1-\theta_{3}\right)}{\gamma\left[\theta_{1} \beta_{1}(\lambda)+1-\theta_{1}\right]}
$$

Now, we calculate

$$
\begin{aligned}
\lim _{\Delta \rightarrow 0} \Phi(z)= & \lim _{\Delta \rightarrow 0}(1-z)\left[\theta_{1} z S_{1}(\bar{a})+(1-\lambda \Delta)\right. \\
& \times\left(1-\theta_{1} z\right)+(1-\lambda \Delta)\left(1-\theta_{3}\right) \\
& \left.\times(1-z)\left(1-S_{1}(\bar{a})\right)\right] \\
& \times\left[\theta_{1} z+(1-\lambda \Delta)\left(1-\theta_{3}\right)\right] \\
& \times(1-z) \gamma S_{1}(\bar{a}) \\
& +(1-\lambda \Delta) \bar{\gamma} z S_{2}\left(\bar{a}+a z+a \theta_{3}(1-z)\right) \\
& -(1-\lambda \Delta) \\
& \times\left[z-\gamma\left(\theta_{1} z^{2}+\theta_{2} z+\theta_{3}\right)\right]^{-1} \gamma \pi_{0} \\
= & (1-z)\left[\theta_{1} z \beta_{1}(\lambda)+1-\theta_{1} z\right. \\
& \left.+\left(1-\theta_{3}\right)(1-z)\left(1-\beta_{1}(\lambda)\right)\right] \\
& \times\left[\left[\theta_{1} z+1-\theta_{3}\right](1-z) \gamma \beta_{1}(\lambda)\right. \\
& +\bar{\gamma} z \beta_{2}\left(\lambda\left(1-\theta_{3}\right)(1-z)\right) \\
& \left.-\left[z-\gamma\left(\theta_{1} z^{2}+\theta_{2} z+\theta_{3}\right)\right]\right]^{-1} \gamma \pi_{0},
\end{aligned}
$$

where

$$
\begin{aligned}
\pi_{0}= & 1-\left(\rho_{1}^{*}+\rho_{2}^{*}\right) \\
= & \frac{1}{\gamma\left[\theta_{1} \beta_{1}(\lambda)+1-\theta_{1}\right]} \\
& \times\left[\left(\theta_{1}+1-\theta_{3}\right) \gamma \beta_{1}(\lambda)-\bar{\gamma}\left(1-\theta_{3}\right) \lambda \mu_{2}^{-1}\right. \\
& \left.-\gamma\left(\theta_{1}-\theta_{3}\right) \gamma\left[\theta_{1} \beta_{1}(\lambda)+1-\theta_{1}\right]\right],
\end{aligned}
$$

which is the generating function of the number of customers in the system $M / G / 1 / \infty$ subject to starting failures and with the service discipline formerly described.

\section{Numerical examples}

In this section, we present some numerical examples to study the effect of the parameters on the main performance characteristics. Of course, in all the examples below, the parametric values are chosen so as to satisfy the stability condition.

We will concentrate on four important performance descriptors: the probability that the system is empty, the mean number of customers in the queue, the mean length of a busy period and the probability that the system is down. To this end, it is assumed that service and repair times follow two different probability distributions with the following generating functions, respectively:

$$
\begin{aligned}
& S_{1}(x)=\frac{x b_{1}}{1-\bar{b}_{1} x}, \\
& S_{2}(x)=\left(\frac{2 x}{3-x}\right)^{n},
\end{aligned}
$$

with $0<b_{1}<1, \bar{b}_{1}=1-b_{1}$.

For the numerical examples we have considered the values of the following probabilities: $\theta_{1}=0.1, \theta_{2}=0.1$, $\theta_{3}=0.8$.

From the stability condition, one finds the value

$$
\begin{aligned}
\gamma> & a \bar{a}\left(1-\theta_{3}\right) \beta_{21}\left\{\left[\theta_{1}+\bar{a}\left(1-\theta_{3}\right)\right] S_{1}(\bar{a})\right. \\
& \left.+a \bar{a}\left(1-\theta_{3}\right) \beta_{21}-\bar{a}\left(\theta_{1}-\theta_{3}\right)\right\}^{-1}=\gamma^{*}
\end{aligned}
$$

Therefore, the system is stable if and only if $\gamma>\gamma^{*}$. Hence the domain of the functions, whose graphs are given below, will be $\left(\gamma^{*}, 1\right]$.

In Figs. 2 and 3, the probability that the system is empty is plotted against the parameter $\gamma$. As we expected, $\pi_{0}$ is an increasing function of the parameter $\gamma$. In Fig. 2 we study the influence of the arrival rate on the probability that the system is empty. Specifically, we present three curves which correspond to $a=0.1, a=0.4, a=0.7$. As expected, $\pi_{0}$ increases with decreasing values of $a$. In Fig. $3, \pi_{0}$ is compared with varying values of the mean repair time. The curves correspond to $\beta_{21}=20,30,40$. It 


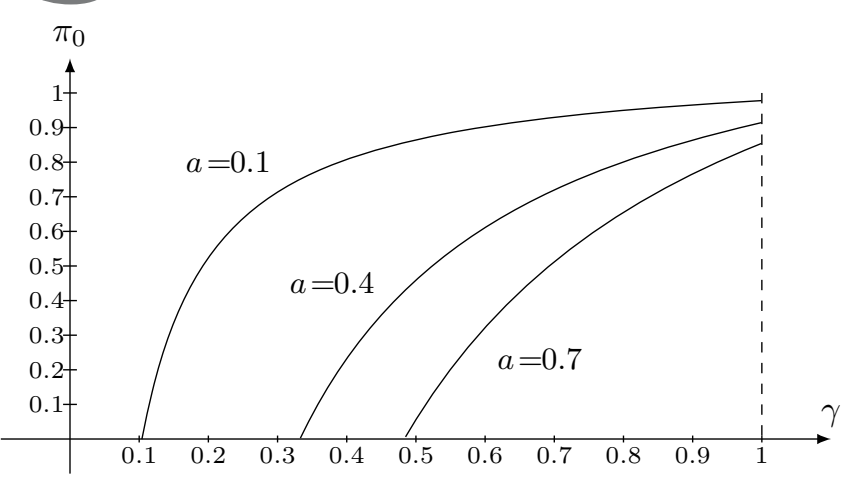

Fig. 2. Probability that the system is empty vs. $\gamma$

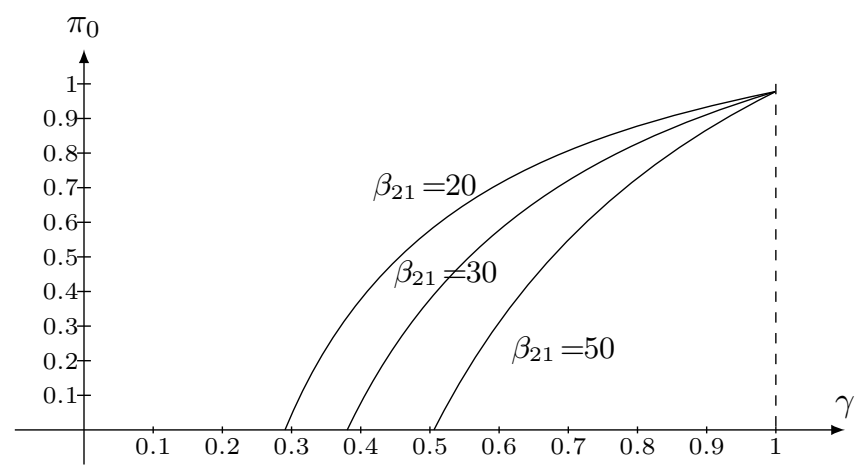

Fig. 3. Probability that the system is empty vs. $\gamma$

can be observed that $\pi_{0}$ decreases with increasing values of the mean repair time, which also agrees with intuition. Moreover, it can be noted that the graphics coincide when $\gamma=1$, as could not be in another way.

Figures 4 and 5 display the effect of the arrival rate and the repair times on the mean queue size. For different choices of the parameters $a$ and $\beta_{21}$, the curves show that $E[N]$ is decreasing as a function of $\gamma$.

These plots also corroborate that the expectation $E[N]$ increases with increasing values of the arrival rate and the repair time.

In Figs. 6 and 7, the mean busy period, $\bar{h}$, is plotted versus $\gamma$ and, as expected, in both graphics, $\bar{h}$ is a decreasing function of $\gamma$, and as intuition says, $\bar{h}$ is increasing with the arrival rate and the repair times.

In Fig. 8 the probability that the server is down is depicted versus $\gamma$. As expected, $\rho_{2}$ is a decreasing function of $\gamma$. It is observed that $\rho_{2}$ is an increasing function of the repair time, which agrees with intuition.

It should be pointed out that the plots in Figs. 3, 5, 7 and 8 are consistent when $\beta_{2,1}$ increases, with intuition

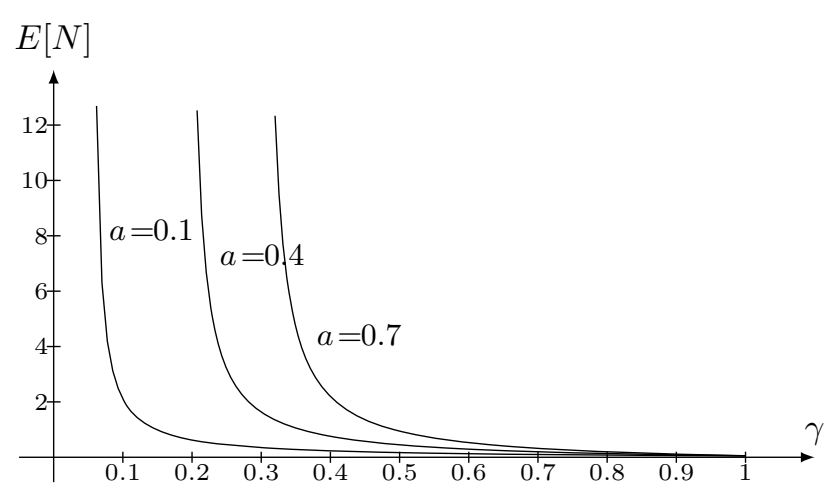

Fig. 4. Mean queue size vs. $\gamma$.

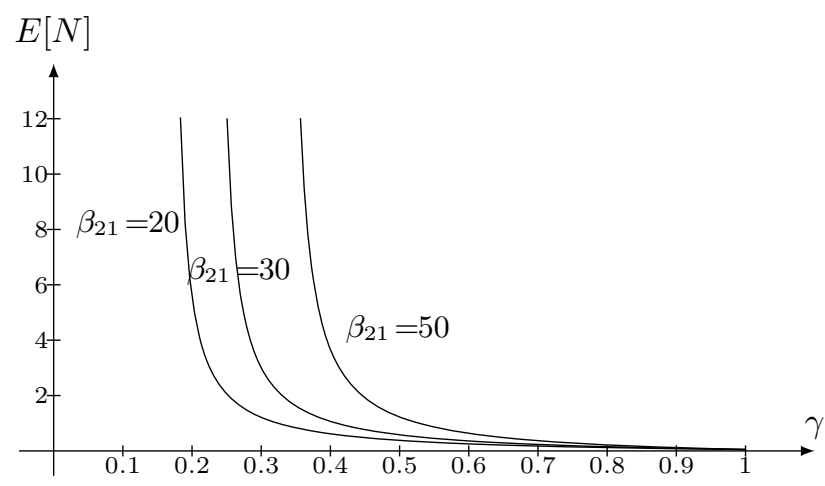

Fig. 5. Mean queue size vs. $\gamma$.

and the limiting result

$$
\lim _{\beta_{2,1} \rightarrow \infty} \gamma^{*}=1 .
$$

Let us also observe that, if $\gamma=1$, the server is reliable and consequently the probability that the server is under repair is equal to zero.

\section{Acknowledgment}

The author thanks the referees for their comments on an earlier version of this paper. This work is supported by the national project TIN2012-39353-C04-01.

\section{References}

Aissani, A. and Artalejo, J. (1998). On the single server retrial queue subject to breakdowns, Queueing Systems 30(3-4): 309-321.

Alfa, A. (2010). Queueing Theory for Telecommunications, 1: Discrete Time Modelling of a Single Node System, Springer, New York, NY. 


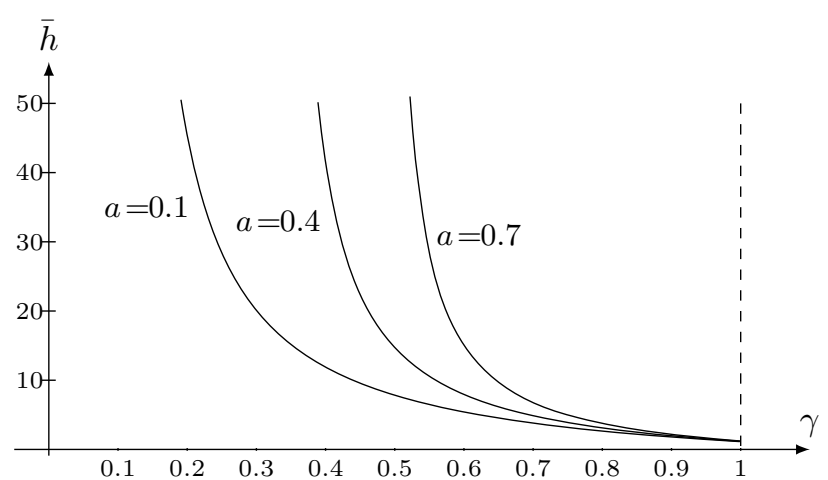

Fig. 6. Mean length of the busy period vs. $\gamma$.

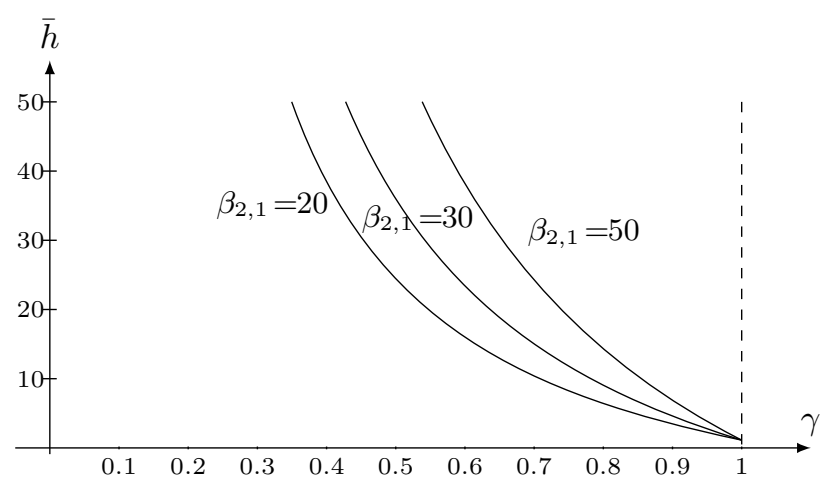

Fig. 7. Mean length of the busy period vs. $\gamma$.

Artalejo, J. (1994). New results in retrial queueing systems with breakdown of the servers, Statistica Neerlandica 48(1): 23-36.

Artalejo, J. (2000). G-networks: A versatile approach for work removal in queueing networks, European Journal of Operational Research 126(2): 233-249.

Atencia, I., Fortes, I., Pechinkin, A. and Sánchez, S. (2013a). A discrete-time queueing system with different types of displacement, 27th European Conference on Modelling and Simulation, Alesund, Norway, pp. 558-564.

Atencia, I., Fortes, I. and Sánchez, S. (2013b). Discrete-time queueing system with expulsions, Communications in Computer and Information Science 356(1): 20-25.

Atencia, I. and Moreno, P. (2004). The discrete-time $\mathrm{Geo} / \mathrm{Geo} / 1$ queue with negative customers and disasters, Computers and Operations Research 31(9): 1537-1548.

Atencia, I. and Moreno, P. (2005). A single-server $G$-queue in discrete-time with geometrical arrival and service process, Performance Evaluation 59(1): 85-97.

Atencia, I. and Pechinkin, A. (2012). A discrete-time queueing system with optional LCFS discipline, Annals Operation Research 202(1): 3-17.

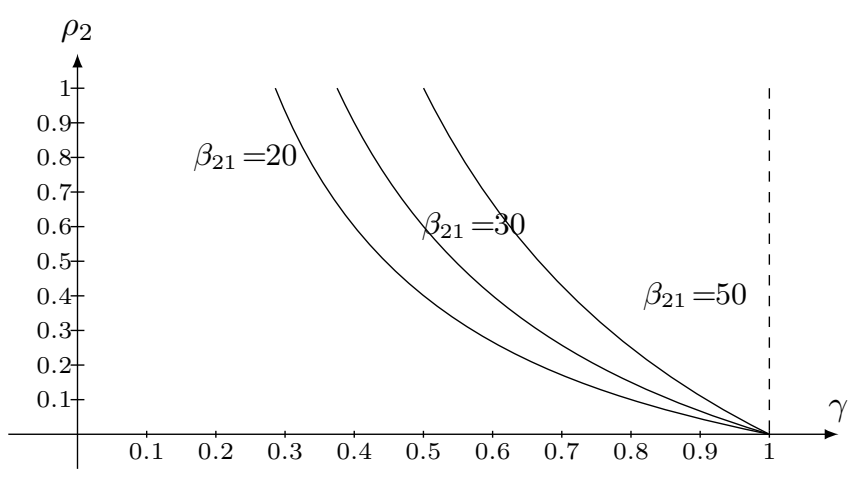

Fig. 8. Probability that the server is down vs. $\gamma$.

Bruneel, H. and Kim, B. (1993). Discrete-time Models for Communication Systems Including ATM, Kluwer Academic Publishers, Boston, MA.

Cascone, A., Manzo, P., Pechinkin, A. and Shorgin, S. (2011). A $G e o_{m} / G / 1 / n$ system with a LIFO discipline without interruptions in the service and with a limitation for the total capacity for the customers, Avtomatika i Telemejanika 1(1): 107-120, (in Russian).

Chao, X., Miyazawa, M. and Pinedo, M. (1999). Queueing Networks: Customers, Signals and Product form Solutions, John Wiley and Sons, Chichester.

Fiems, D., Steyaert, B. and Bruneel, H. (2002). Randomly interrupted $G I / G / 1$ queues: Service strategies and stability issues, Annals of Operations Research 112(1-4): 171-183.

Fiems, D., Steyaert, B. and Bruneel, H. (2004). Discrete-time queues with generally distributed service times and renewal-type server interruptions, Performance Evaluation 55(3-4): 277-298.

Gelenbe, E. and Label, A. (1998). G-networks with multiple classes of signals and positive customers, European Journal of Operational Research 108(2): 293-305.

Gravey, A. and Hébuterne, G. (1992). Simultaneity in discrete-time single server queues with Bernoulli inputs, Performance Evaluation 14(2): 123-131.

Harrison, P.G., Patel, N.M. and Pitel, E. (2000). Reliability modelling using g-queues, European Journal of Operational Research 126(2): 273-287.

Hunter, J. (1983). Mathematical Techniques of Applied Probability, Academic Press, New York, NY.

Kendall, D. (1951a). Some problems in the theory of queues, Royal Statistical Society Series 13(2): 151-185.

Kendall, D. (1951b). Stochastic processes occurring in the theory of queues and their analysis by the method of imbedded Markov chains, Annals of Mathematical Statistics 24(3): 338-354.

Kleinrock, L. (1976). Queueing Systems, Vol. 2, John Wiley and Sons, New York, NY. 
Krishna Kumar, B., Pavai Madheswari, S. and Vijayakumar, A. (2002). The $M / G / 1$ retrial queue with feedback and starting failures, Applied Mathematical Modelling 26(11): 1057-1075.

Krishnamoorthy, A., Pramod, P. and Deepak, T. (2009). On a queue with interruptions and repeat or resumption of service, Nonlinear Analysis: Theory, Methods \& Applications 71(12): 1673-1683.

Kulkarni, V. and Choi, B. (1990). Retrial queues with server subject to breakdowns and repairs, Queueing Systems 7(2): 191-208.

Meisling, T. (1958). Discrete time queueing theory, Operations Research 6(1): 96-105.

Morozov, E., Fiems, D. and Bruneel, H. (2011). Stability analysis of multiserver discrete-time queueing systems with renewal-type server interruptions, Performance Evaluation 68(12): 1261-1275.

Oniszczuk, W. (2009). Semi-Markov-based approach for the analysis of open tandem networks with blocking and truncation, International Journal of Applied Mathematics and Computer Science 19(1): 151-163, DOI: 10.2478/v10006-009-0014-6.

Park, H.M., Yang, W.S. and Chae, K.C. (2009). The Geo/G/1 queue with negative customers and disasters, Stochastic Models 25(4): 673-688.

Pechinkin, A. and Shorgin, S. (2008). A $G e o / G / 1 / \infty$ system with a non-standard discipline for the service, Informatics and Its Applications 2(1): 55-62, (in Russian).

Pechinkin, A. and Svischeva, T. (2004). The stationary state probability in the $B M A P / G / 1 / r$ queueing system with inverse discipline and probabilistic priority, Transactions of the XXIV International Seminar on Stability Problems for Stochastic Models, Jurmala, Latvia, pp. 141-174.
Piórkowski, A. and Werewka, J. (2010). Minimization of the total completion time for asynchronous transmission in a packet data-transmission system, International Journal of Applied Mathematics and Computer Science 20(2): 391-400, DOI: 10.2478/v10006-010-0029-z.

Takagi, H. (1993). Queueing Analysis: A Foundation of Performance Evaluation: Discrete-Time Systems, North-Holland, Amsterdam.

Vinck, B. and Bruneel, H. (2006). System delay versus system content for discrete-time queueing systems subject to server interruptions, European Journal of Operational Research 175(1): 362-375.

White, H. and Christie, L. (1958). Queuing with preemptive priorities or with breakdown, Operations Research 6(1): 79-95.

Yang, T. and Li, H. (1994). The $M / G / 1$ retrial queue with the server subject to starting failures, Queueing Systems 16(1-2): 83-96.

Yang, T. and Li, H. (1995). On the steady-state queue size distribution of the discrete-time $G e o / G / 1$ queue with repeated customers, Queueing Systems 21(1-2): 199-215.

Ivan Atencia graduated in 1997 and obtained his Ph.D. in mathematics in 2000 from Malaga University, Spain. During this period he collaborated with the Department of Probability Theory and Mathematical Statistics of the Peoples' Friendship University of Russia, Moscow. At present, he is an associate professor in the Department of Applied Mathematics at Malaga University and actively collaborates with the Institute of Informatics Problems of the Russian Academy of Sciences. His research interests include queueing theory and stochastic modeling of communication systems.
Received: 19 August 2013

Revised: 30 January 2014

Re-revised: 26 March 2014 